\title{
Compressive Spectrum Sensing Using a Bandpass Sampling Architecture
}

\author{
Linda Bai, Student Member, IEEE \& Sumit Roy, Fellow, IEEE
}

\begin{abstract}
Fast and reliable detection of available channels (i.e., those temporarily unoccupied by primary users) is a fundamental problem in the context of emerging cognitive radio networks, without an adequate solution. The (mean) time to detect idle channels is governed by the front-end bandwidth to be searched for a given resolution bandwidth. Homodyne receiver architectures with a wideband $\mathrm{RF}$ front-end followed by suitable channelization and digital signal processing algorithms, are consistent with speedier detection, but also imply the need for very high speed analog-to-digital converters (ADCs) that are impractical and/or costly. On the other hand, traditional heterodyne receiver architectures consist of analog band-select filtering followed by down-conversion that require much lower rate ADCs, but at the expense of significant scanning operation steps that constitute a roadblock to lowering the scan duration. In summary, neither architecture provides a satisfactory solution to the goal of (near) real-time wideband spectrum sensing.

In this work, we propose a new compressive spectrum sensing architecture based on the principle of under-sampling (or bandpass sampling) that provides a middle ground between the above choices, i.e., our approach requires modest ADC sampling rates and yet achieves fast spectrum scanning. Compared to other compressive spectrum sensing architectures, the proposed method does not require a high-speed Nyquist rate analog component. A performance model for the scanning duration is developed based on the mean time to detect all idle channels. Numerical results show that this scheme provides significantly faster idle channel detection than the conventional serial search scheme with a heterodyne architecture.
\end{abstract}

Index Terms-Compressive Sensing, Cognitive Radio, Wideband Spectrum Sensing, Transceiver Architectures

\section{INTRODUCTION}

Continued proliferation of wireless services has led to renewed scrutiny of spectrum utilization. In particular, several licensed bands, e.g., land mobile and amateur radio, have been shown to be under-utilized with occupancy rates between $15 \%$ to $85 \%$ [1]. Cognitive radios - enabled by emerging software defined transceiver architectures - is an approach for enhanced spectrum utilization, by allowing unlicensed users to opportunistically access licensed bands, when the latter are unused. The unlicensed or secondary users must sense the spectrum to detect idle channels (free of incumbents or primary users) and are allowed to utilize the idle channels, provided they do

The authors are with the Department of Electrical Engineering, University of Washington, Seattle, WA 98195, USA (e-mail: lyb3@uw.edu; roy@ee.washington.edu).This work was supported in part by the National Science Foundation Graduate Research Fellowship to the 1st author under Grant No. DGE-0718124. Copyright (c) 2012 IEEE. Personal use of this material is permitted. However, permission to use this material for any other purposes must be obtained from the IEEE by sending an email to pubspermissions@ieee.org. not interfere with the licensed users, i.e., vacate the channel when a primary user transmission is detected.

Therefore, one of the primary requirements for cognitive radio is fast and effective detection of idle channels to exploit the available secondary network capacity [1]. Due to the large bandwidth (for example, up to $10 \mathrm{GHz}$ ) that needs to be sensed, speedy idle channel detection is a challenging task. Depending on the bandwidth needs required by the applications, secondary users may seek to detect one idle channel or all available idle channels. For detection of one idle channel, the receiver stops searching as soon as it detects the first idle channel, whereas in the latter, the receiver will search the status of all channels in the spectrum of interest. In this paper, we focus on the second scenario, consistent with the desire to maximize secondary throughput.

Assume that the entire (one-sided) spectrum of bandwidth $W=N W_{c}$ is channelized into an $N$-set of contiguous frequency domain channels of bandwidth $W_{c}$ each. The choice of receiver architecture is a major factor that influences the mean detection time of all the idle channels among these $N$ channels. The heterodyne architecture is the most popular legacy architecture due to its high selectivity and sensitivity [2]. Heterodyne receivers use analog bandpass filters for selecting a $W_{c}$-width channel as shown in Fig.1. The output is then down-converted to baseband and fed into an analog-todigital converter (ADC) [3]. The ADC samples at $f_{s}=W_{c}$ (to produce the complex baseband signal), at a rate which is much smaller than the total signal bandwidth $N W_{c}$. The receiver decides the status of the selected channel using one of several standard detection algorithms described in [4]. After the status of the selected channel is determined, the receiver switches to the next channel in sequence. A conventional serial search scheme (implying a sweep or step-through of the LO frequency) is used to determine the occupancy status of all the $N$ channels.

For the heterodyne receiver architecture, the time to detect idle channels has two components - a) the integration time $T_{i}$ required by the detection algorithm once a channel has been selected for investigation; and $b$ ) the duration for switching to a new channel $T_{s w}$ during scanning, that is largely determined by the design/operation of the LO [5]. Denote $N_{i}$ as the number of samples required for meeting desired detection accuracy in each channel for a given detection algorithm, then $T_{i} \approx N_{i} / W_{c}$. The mean detection time for the heterodyne receiver to determine status for all channels is thus $T_{d, \text { serial }}=N\left(T_{i}+T_{s w}\right)$. For example, for $W_{c}=200 \mathrm{kHz}$, $N=1024, N_{i}=1000$, and $T_{s w} \approx 50 \mu \mathrm{s}$ as in [6], then $T_{i}=5 \mathrm{~ms}$, and $T_{d, \text { serial }} \approx \mathbf{5 . 1 7} \mathrm{sec}$. 

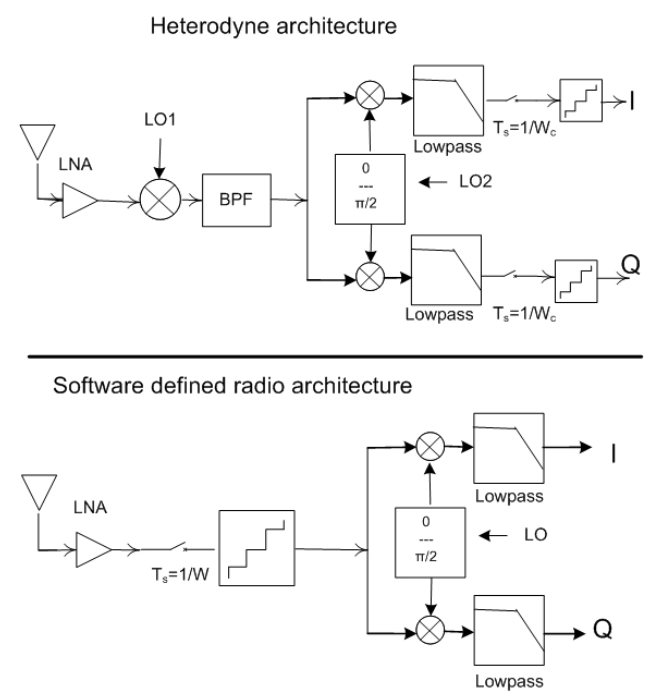

Fig. 1. Block diagram of the heterodyne and homodyne receiver architectures

On the other hand, in homodyne receiver architectures such as that in Fig.1, the wideband RF received signal is sampled directly by an ADC, followed by subsequent digital signal processing for channelization and determination of channel status. For digital signal processing, the power spectrum is obtained by squared magnitude of the $N$-point FFT for the wideband signal. Each bin in the power spectrum represents the estimated power in the corresponding channel, and is compared with a power threshold to determine the status of the channel [7]. An estimate of the (mean) time to determine status of all $N$ channels is obtained by noting that the total number of samples needed is $N \cdot N_{i}$, when $N_{i}$ samples are required for each channel. Since the sampling rate of the ADC for the complex baseband signal is $N \cdot W_{c}$, the acquisition time is $\left(N \cdot N_{i}\right) /\left(N \cdot W_{c}\right)=N_{i} / W_{c}$. Note that signals in all the $N$ channels are acquired in parallel and hence the analog circuit switching time $T_{s w}$ is avoided, yielding an acquisition time that is independent of $N$. Denote $T_{d s p}$ as the latency due to the subsequent signal processing operations such as the FFT in generating the spectrogram. Hence the time to determine status of all channels for such a homodyne architecture is $T_{d, s d r}=N_{i} / W_{c}+T_{d s p}$. For the spectrum sensing task above, the detection time for all the idle channels with the software defined radio architecture (i.e., the homodyne architecture) is approximately $\mathbf{3 1} \mathrm{ms}$, with $T_{d s p}=N_{i} \cdot 26 \mu \mathrm{s}$, where $26 \mu \mathrm{s}$ is the typical processing time for a 1024-point FFT [8]. Thus, software defined radio (SDR) based transceivers can potentially achieve faster detection by two orders of magnitude, at the expense of an ultra-fast (multi-GHz) ADC for Nyquist sampling of the wideband signal, that considerably increases the power consumption and IC complexity [9].

In this work, we unveil a new, compressive architecture based on sub-Nyquist ${ }^{1}$ sampling ADCs. Our proposal thus treads a middle ground by combining the strengths of the two architectures, in terms of achieving fast detection, while

\footnotetext{
${ }^{1}$ The sampling is sub-Nyquist relative to the rate required to sample the (bandpass) spectra treated as a low pass signal.
}

requiring a lower rate $\mathrm{ADC}$.

\section{RELATED WORKS}

\section{A. Brief Review of Compressive Sensing}

Compressive sensing (CS) is a well-known technique to reconstruct a sparse $N$-dimensional signal vector $\mathrm{x}$ from an $M$-dimensional representation $\mathbf{y}=\boldsymbol{\Phi} \mathbf{\Psi}_{\mathbf{X}}(M \ll N)[10]$, where the $N \times N$ matrix $\boldsymbol{\Psi}$ is the sparsifying basis. In the spectrum sensing scenario, $\Psi$ is the Inverse Fourier Transform matrix, and $\mathbf{x}$ represents the amplitude spectra in the frequency domain. There exists a mature literature regarding appropriate choices of the observation matrix $\boldsymbol{\Phi}$, whose elements are typically i.i.d Gaussian or Bernoulli variables [10]. An appropriate choice of $\boldsymbol{\Phi}$ must satisfy the Restricted Isometry Property (RIP), formally defined as follows [11],

Definition 1: Restricted Isometry Property A matrix $\boldsymbol{\Phi}$ satisfies RIP with parameter $\left(k, \delta_{k}\right)$ if

$$
\left(1-\delta_{k}\right)\|\mathbf{x}\|_{2}^{2} \leq\|\mathbf{\Phi} \mathbf{x}\|_{2}^{2} \leq\left(1+\delta_{k}\right)\|\mathbf{x}\|_{2}^{2}
$$

for all $k$-sparse vectors $\mathbf{x}$, where $k$-sparsity means there are at most $k$ non-zero elements in the vector $\mathbf{x}$.

Given $\boldsymbol{\Phi}$ satisfies RIP with $\left(2 k, \delta_{2 k}\right)$ and $0<\delta_{2 k}<\sqrt{2}-$ 1 , the $k$-sparse vector $\mathbf{x}$ is reconstructed using

$$
\begin{array}{r}
\text { minimize }\|\mathbf{x}\|_{1} \\
\text { subject to } \mathbf{y}=\mathbf{\Phi} \mathbf{\Psi} \mathbf{x}
\end{array}
$$

Of late, applications of CS to spectrum estimation in cognitive radio has appeared in [12], [13], [14], and [15]. Most of the previous works focus on algorithm development for reconstruction, such as [12] and [14], while in this paper, we emphasize the architectural aspects of compressive detectors and its impact on performance. Therefore, for baseline, we compared our architecture performance with the preferred architectures in prior art. Various receiver architectures for spectrum sensing with sub-Nyquist sampling have been proposed based on compressive sensing theory. These architectures can be divided into two classes: non-uniform sampler and random pre-integrator [16]. Both receiver architectures consist of subNyquist sampling ADCs to obtain an observation matrix $\boldsymbol{\Phi}$ that satisfies RIP. Nevertheless, these sub-Nyquist sampling approaches based on CS can provide accurate results only if most of the channels in the spectrum are idle, i.e., $\bar{p} \approx 1$, where $\bar{p}$ denotes the average probability of a channel being idle. If the number of occupied channels is close to the number of idle channels, i.e., $\bar{p} \approx 0.5$, current CS-based reconstruction approaches fail to provide accurate channel status [16]. In addition, compressive detection incurs high computational complexity to solve (2), leading to extended signal processing time. Furthermore, although the sampler in the ADC operates at a sub-Nyquist rate, the detector actually requires Nyquist rate analog components as discussed in Sec. II-B [17]. For the non-uniform sampler, a Nyquist rate clock is required for the shifters; for the random pre-integrator, a Nyquist rate random generator is required. 


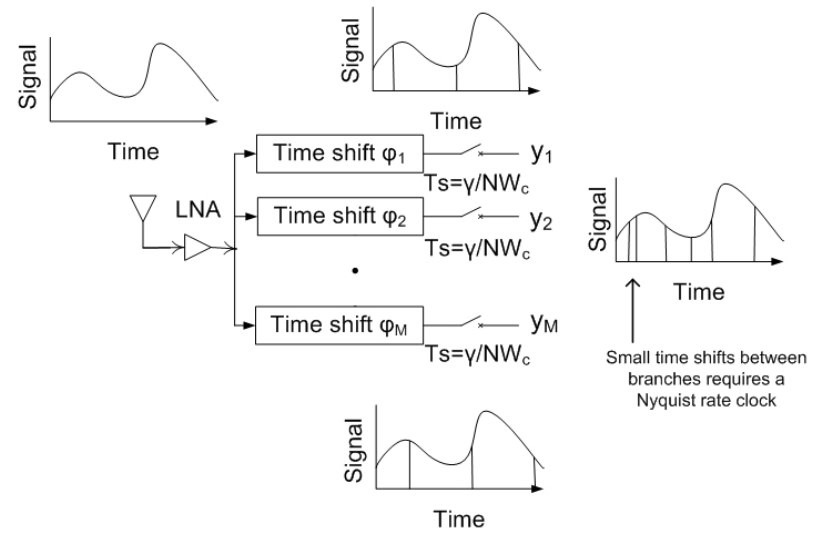

Fig. 2. Architecture of a non-uniform sampler

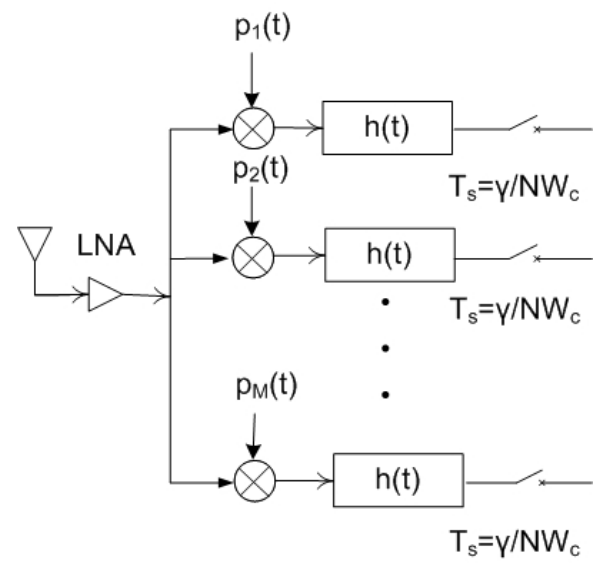

Fig. 3. Architecture of a random pre-integrator

B. Non-uniform Sampling and Random Pre-integration: Compressive Detection Approaches

The architecture of a non-uniform sampler is shown in Fig.2 [18][19] where the wideband input signal is sampled at a sub-Nyquist rate $f_{s}=\frac{1}{T_{s}}=\frac{N W_{c}}{\gamma}$ by $M$ branches, each with a different time shift misalignment $\phi_{i}, i=1, \ldots, M$, where $\gamma>1$ is the sub-sampling factor. In terms of analog implementation, the non-uniform sampler operates at a subNyquist rate, but requires a Nyquist rate clock to synchronize the shifters on each branch. On the other hand, an example of a random pre-integrator architecture is shown in Fig. 3 [17]. In a random pre-integrator [20][21], a demodulator multiplies the received (analog) signal with a random chipping sequence; the resulting product is integrated over a symbol duration and sampled at a sub-Nyquist rate on each of the $M$ branches. The chipping sequence generator is required to work at greater or equal to the Nyquist rate to produce a $\boldsymbol{\Phi}$ with i.i.d. Bernoulli or Gaussian entries.

Denote the signal processing time for (2) as $T_{l 1}$, then the mean detection time for the non-uniform sampler is

$$
T_{d, C S}=\left(N \cdot N_{i}\right) /\left(N \cdot W_{c}\right)+N_{i} T_{l 1}=N_{i} / W_{c}+N_{i} T_{l 1}
$$

where $T_{l 1}$ is determined by the computational complexity of the signal processing algorithms. Using the non-uniform sampling architecture, the complexity for solving (2) is $O\left(M^{2} N^{1.5}\right)$ [22]. In [23], as $M \sim O((N-\bar{p} N) \log (1 /(1-$ $\bar{p}))), T_{l 1} \sim O\left(((1-\bar{p}) N)^{3}\right)$. When $N_{i}$ spectrum snapshots are reconstructed and averaged to generate the power spectrum, the digital signal processing time is $O\left(((1-\bar{p}) N)^{3} N_{i}\right)$ for both the random pre-integrator and non-uniform sampling architectures. Note that (2) requires the sensing matrix $\boldsymbol{\Phi} \boldsymbol{\Psi}$ to be stored on-chip, yielding a memory requirement of $O(M N)$. However, the storage required for the non-uniform sampler is $O(M)$. Since $\boldsymbol{\Phi} \boldsymbol{\Psi}$ is a subset of the IDFT matrix, only the indices for the $M$ chosen rows need to be stored, and $\boldsymbol{\Phi} \Psi$ can be reconstructed using only the indices.

\section{Contributions}

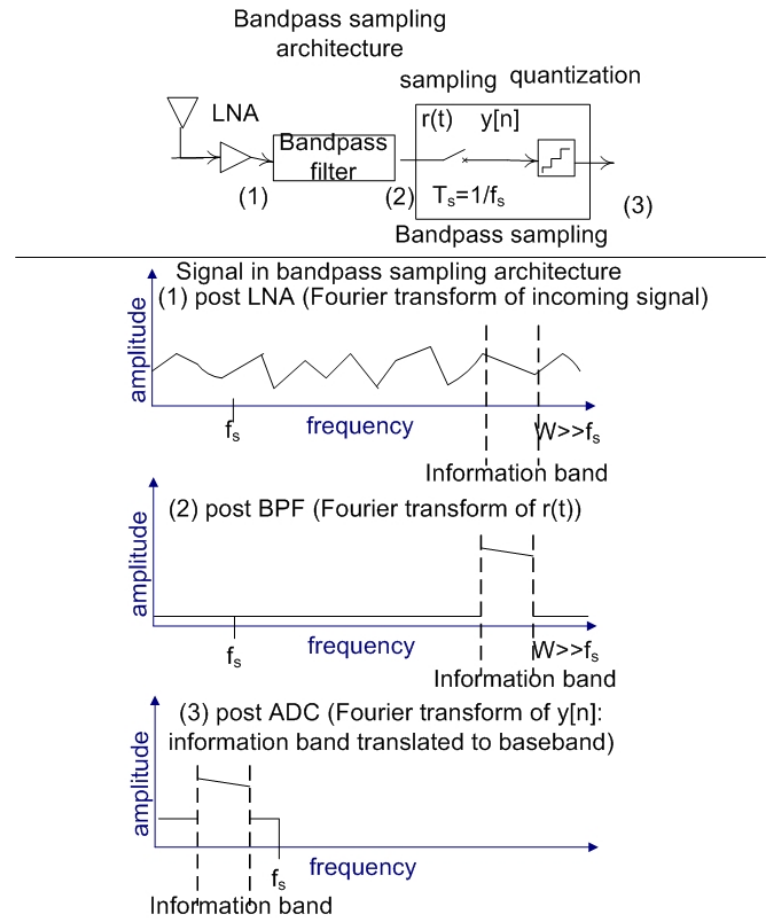

Fig. 4. Block diagram of a bandpass sampling architecture for direct downconversion

Our work is a synthesis of the notion of Compressive Sensing within a bandpass sampling architecture. The traditional bandpass sampling receiver chain is shown in Fig.4, where the complex baseband signal $r(t)$ is sampled at a sub-Nyquist rate $f_{s}=\frac{1}{T_{s}}=\frac{N W_{c}}{\gamma}$ to obtain $y[n]=r\left(n T_{s}\right)$, where $\gamma>1$ is the sub-sampling factor [24]. Note that for compactness, only the analytic signal that occupies the positive frequency spectrum, is shown. This architecture achieves frequency downconversion by undersampling $r(t)$ (viewed as a wideband lowpass signal), where the sampling rate is determined by the bandwidth of the desired bandpass signal (i.e., conforming to the bandpass Nyquist sampling theorem) instead of the carrier frequency [25]. Owing to the reduction of the sampling rate, bandpass sampling simplifies the design of the local oscillator (LO), leading to better performance than a mixer for downconversion [3]. 
In Sec.III, a spectrum sensing scheme with a bandpass sampling architecture is proposed. Here, the entire spectrum (in principle) can be sensed simultaneously, reducing the need for LO scanning. The proposed architecture switches between different (sub-Nyquist) sampling rates to obtain an observation matrix that satisfies RIP; no Nyquist-rate analog component is needed. Instead of reconstructing each snapshot of the amplitude spectra, the power spectrum is reconstructed directly by $l_{1}$ minimization, mitigating the delay of intense signal processing.

In Sec.IV, the performance of the proposed architecture is analyzed. The mean detection time, the computational complexity, and the required memory for storing the observation matrix are compared with the non-uniform sampler and the random pre-integrator. In addition, the detection rate and the false alarm rate for the proposed scheme is analyzed. A low detection rate will cause interference to primary users, while a low false alarm rate is necessary for a high spectral utilization rate. Numerical results for the mean detection time are presented as a function of the number of channels to be sensed. Impact of Signal-to-Noise Ratio (SNR) and $\bar{p}$ is explored. Finally, in Sec.V, the proposed scheme is validated using real-world spectrum measurements obtained in Aachen, Germany.

\section{SYSTEM DESCRIPTION}

\section{A. System Model}

Assume that the band of interest consists of $N$ equi-spaced channels; the central frequency of the $i^{\text {th }}$ channel is $f_{i}=$ $i W_{c}-\frac{W_{c}}{2}$, where $W_{c}$ is the bandwidth for each channel. A binary variable, $O_{i}$ is used to denote the status of the $i^{t h}$ channel, where $O_{i}=0(1)$ means the channel is busy (idle). We invoke an independent Bernoulli model for channel status, that is supported by real-world data as described in [26], i.e.,

$$
\operatorname{Pr}\left(O_{i}=1\right)=p_{i}, i=1, \ldots, N
$$

Thus, the average signal power $\bar{P}_{s, i}$ in the $i^{t h}$ channel is described as a Bernoulli variable, with $\operatorname{Pr}\left(\bar{P}_{s, i}=0\right)=p_{i}$ and $\operatorname{Pr}\left(\bar{P}_{s, i}=\bar{P}_{r, i}\right)=1-p_{i}$, where $p_{i}$ is the probability of the $i^{t h}$ channel being idle. $\bar{P}_{r, i}$ is the signal power in the $i^{t h}$ channel at the receiver when the channel is occupied by the primary user. The average idle channel probability over the entire band is given by $\bar{p}=\frac{1}{N} \sum_{i=1}^{N} p_{i}$.

\section{B. Compressive sensing architecture}

Denote the received signal as $r(t)$ whose bandwidth $W=$ $N W_{c}$. The discrete-time Fourier transform $Y(f)$ of the signal $y[n]$ sampled at the rate $f_{s}$ is related to $R(f)$, the Fourier transform of $r(t)$, by the well-known result

$$
Y(f)=\frac{1}{T_{s}} \sum_{k=-\infty}^{\infty} R\left(f-k f_{s}\right)
$$

An example is illustrated in Fig.5 where the complex baseband signal $r(t)$, comprising of 12 channels each of bandwidth $W_{c}$, is sampled at a (sub-Nyquist) rate of $f_{s}=$

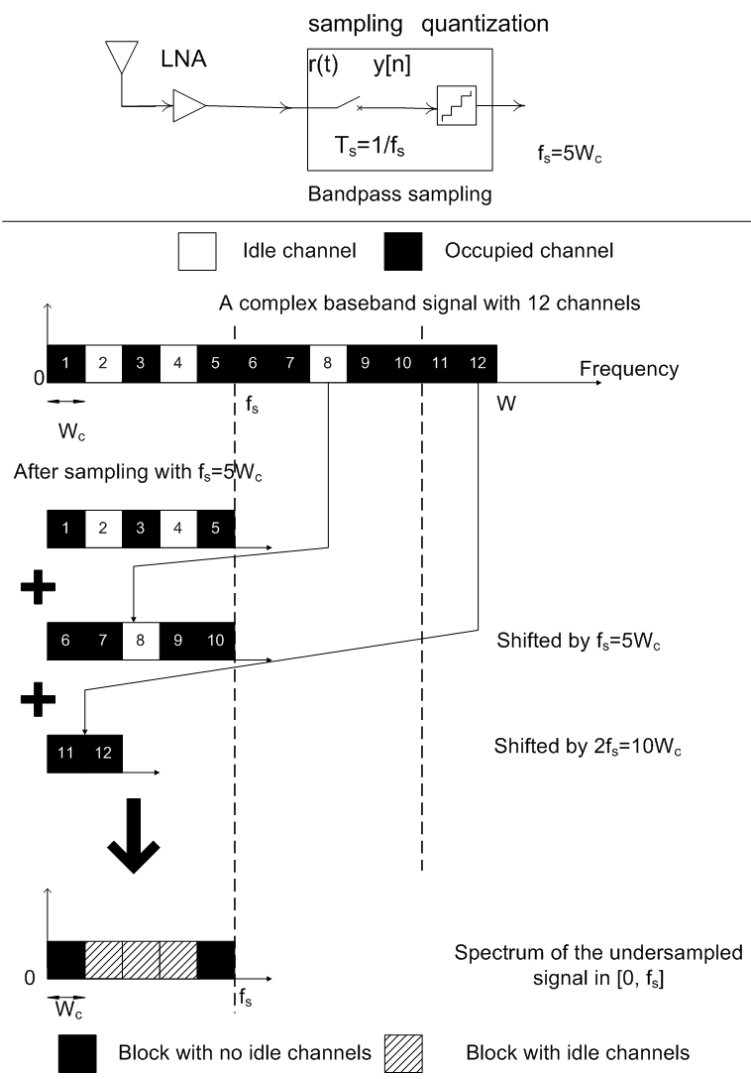

Fig. 5. Sub-Nyquist rate sampling: a signal with 12 channels sampled at $5 W_{c}$

$5 W_{c}$. As shown in Fig.5, aliasing occurs in the spectra of the sampled signal due to the sub-Nyquist sampling rate; the sixth channel folds back onto the first channel, since $f_{6}-f_{s}=\frac{11}{2} W_{c}-5 W_{c}=\frac{1}{2} W_{c}=f_{1}$. Clearly, when a signal is sub-sampled with rate $f_{s}$, all frequency components $\left\{f_{i} \pm k f_{s}\right\}, k=0,1, \ldots$ present in the signal alias back onto the same frequency $f_{i} \in\left[0, f_{s}\right]$, where $\left[0, f_{s}\right]$ is the first Nyquist zone. Letting $f_{s}=\frac{N}{\gamma} W_{c}$, where $\frac{N}{\gamma}$ is an integer, the relationship between $Y_{i}, i=1, \ldots, \frac{N}{\gamma}$, (the Fourier coefficients of $y[n]$ on the interval $\left[0, f_{s}\right]$ ) and $R_{i}, i=1, \ldots, N$, (the Fourier coefficients of $r[n]$ sampled at Nyquist rate $N W_{c}$ on the interval $\left.\left[0, N W_{c}\right]\right)$ is

$$
\mathbf{Y}=\left[\begin{array}{c}
Y_{1} \\
\vdots \\
Y_{\frac{N}{\gamma}}
\end{array}\right]=\frac{1}{\gamma} \boldsymbol{\Phi}\left[\begin{array}{c}
R_{1} \\
\vdots \\
R_{N}
\end{array}\right]=\frac{1}{\gamma} \mathbf{\Phi R}
$$

where the $\frac{N}{\gamma} \times N$ matrix

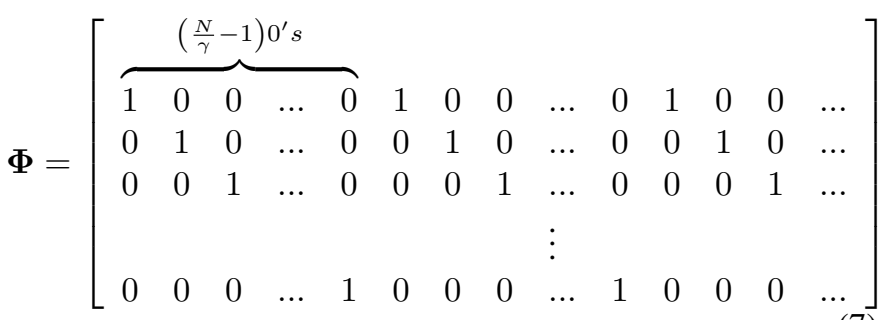


For example, let $N=10$, and $\gamma=2$. Then

$$
\begin{aligned}
Y_{1} & =\sum_{i=0}^{4} r\left(i / f_{s}\right)=\sum_{i=0}^{4} r\left(i \gamma / N W_{c}\right) \\
& =\sum_{i=0}^{4} r\left(2 i / N W_{c}\right)
\end{aligned}
$$

Since

$$
R_{1}=\sum_{i=0}^{9} r\left(i / N W_{c}\right)
$$

and

$$
\begin{aligned}
R_{5} & =\sum_{i=0}^{9} r\left(i / N W_{c}\right) e^{\frac{j 2 \pi}{10} 5 i} \\
& =\sum_{i=0}^{4} r\left(2 i / N W_{c}\right)-\sum_{i=0}^{4} r\left((2 i+1) / N W_{c}\right)
\end{aligned}
$$

it follows that $Y_{1}=\frac{1}{2}\left(R_{1}+R_{5}\right)$. Therefore, each element in $\mathbf{Y}$ is the average of the amplitude spectra over a block of $\gamma$ channels in $\mathbf{R}$. Let $\mathbf{R}=\mathbf{S}+\mathbf{V}$, where $\mathbf{S}=\left[S_{1}, \ldots, S_{N}\right]^{T}$ is the signal component and $\mathbf{V}=\left[V_{1}, \ldots, V_{N}\right]^{T}$ is the noise component, $E\left(\left|S_{i}\right|^{2}\right)=\bar{P}_{s, i}$, and $E\left(\left|V_{i}\right|^{2}\right)=\bar{P}_{v, i}$, where $\bar{P}_{s, i}\left(\bar{P}_{v, i}\right)$ is the average signal (noise) power in the $i^{\text {th }}$ channel, respectively. Then (6) can be written as

$$
\mathbf{Y}=\frac{1}{\gamma} \boldsymbol{\Phi}(\mathbf{S}+\mathbf{V})
$$

The sequences $S_{i}, V_{i}$ are assumed to consist of independent variables (corresponding to different channels) that are mutually independent. It is natural that $E\left(V_{i}\right)=0$ and for symmetric signal constellations,

$$
E\left(S_{i}\right)=0
$$

as well. Then $R_{i}$ and $R_{j}$ are independent, $\forall i \neq j$, and $E\left(R_{i}\right)=E\left(S_{i}\right)+E\left(V_{i}\right)=0, E\left(\left|R_{i}\right|^{2}\right)=\bar{P}_{s, i}+\bar{P}_{v, i}$. Typically the time samples $r[n]$ are complex Gaussian variables, whose Fourier coefficients $R_{i} \sim C N\left(0,\left(\bar{P}_{s, i}+\bar{P}_{v, i}\right) / 2\right), i=$ $1, \ldots, N$ [27]. The expectation of the power in the $m^{\text {th }}$ block is

$$
\begin{aligned}
E\left(Y_{m} Y_{m}^{*}\right) & =E\left(\frac{1}{\gamma^{2}}\left|\sum_{j^{\prime}=1}^{\gamma} R_{j^{\prime}}\right|^{2}\right) \\
& =\frac{1}{\gamma^{2}} E\left(\sum_{j^{\prime}=1}^{\gamma}\left|R_{j^{\prime}}\right|^{2}+\sum_{j_{1}^{\prime} \neq j_{2}^{\prime}} R_{j_{1}^{\prime}} R_{j_{2}^{\prime}}^{*}\right) \\
& =\frac{1}{\gamma^{2}} \sum_{j^{\prime}=1}^{\gamma} \bar{P}_{s, j^{\prime}}+\bar{P}_{v, j^{\prime}}
\end{aligned}
$$

where $\bar{P}_{s, j^{\prime}}$ and $\bar{P}_{v, j^{\prime}}$ are the average signal power and noise power in the $j^{t h}$ channel in the $m^{t h}$ block, respectively.

We propose to use the bandpass sampling architecture shown in Fig.5 to sense the spectrum $K$ times, each for a different sub-Nyquist sampling rate, in order to obtain a $\boldsymbol{\Phi}$ that satisfies the RIP. An equivalent architecture is shown in Fig. 6 where the incoming signal is fed into $K$ branches. The $k^{t h}$ branch consists of an ADC sampling at $\frac{N W_{c}}{\gamma_{k}}$, followed by a square-law device and an integrator. Note that instead of the multi-branch architecture, a single branch detector as in Fig.5 that sequentially switches between different sampling rates is also feasible. However, the resulting mean detection times are different. Clearly, the multi-branch architecture yields a reduced sensing duration due to the inherent parallelism, at the cost of chip complexity. Our analysis for mean detection time in Sec.IV is based on the single branch architecture.

The measurement $\mathbf{Y}_{k, n}$ for the $k^{t h}$ sampling rate and the $n^{\text {th }}$ snapshot is used to create the spectrogram, denoted by a $\frac{N}{\gamma_{k}} \times 1$ vector $\mathbf{w}_{k, n}$, where $\mathbf{w}_{k, n}(i)=\gamma_{k}^{2}\left|\mathbf{Y}_{k, n}(i)\right|^{2}, n=$ $1, \ldots, N_{i}$, are i.i.d corresponding to different snapshots. Now

$$
E\left(\mathbf{w}_{k, n}\right)=\boldsymbol{\Phi}_{k}\left[\begin{array}{c}
\bar{P}_{s, 1}+\bar{P}_{v, 1} \\
\vdots \\
\bar{P}_{s, N}+\bar{P}_{v, N}
\end{array}\right]
$$

where $\boldsymbol{\Phi}_{k}$ is constructed according to (7),

$$
\begin{array}{cc} 
& \operatorname{Var}\left(\mathbf{w}_{k, n}(i)\right) \\
= & E\left(\left(\mathbf{w}_{k, n}(i)\right)^{2}\right)-\left(E\left(\mathbf{w}_{k, n}(i)\right)\right)^{2} \\
= & E\left(\left|\sum_{j^{\prime}=1}^{\gamma_{k}} R_{j^{\prime}}\right|^{4}\right)-\left(\sum_{j^{\prime}=1}^{\gamma_{k}}\left(\bar{P}_{s, j^{\prime}}+\bar{P}_{v, j^{\prime}}\right)\right)^{2} \\
= & \left(\sum_{j^{\prime}=1}^{\gamma_{k}}\left(\bar{P}_{s, j^{\prime}}+\bar{P}_{v, j^{\prime}}\right)\right)^{2}
\end{array}
$$

For $i_{1} \neq i_{2}$,

$$
\begin{array}{cc} 
& \operatorname{Cov}\left(\mathbf{w}_{k, n}\left(i_{1}\right), \mathbf{w}_{k, n}\left(i_{2}\right)\right) \\
= & E\left(\left(\mathbf{w}_{k, n}\left(i_{1}\right)\right)\left(\mathbf{w}_{k, n}\left(i_{2}\right)\right)\right) \\
= & -\left(E\left(\mathbf{w}_{k, n}\left(i_{1}\right)\right)\right)\left(E\left(\mathbf{w}_{k, n}\left(i_{2}\right)\right)\right) \\
= & 0
\end{array}
$$

The averaged power over $N_{i}$ snapshots

$$
\overline{\mathbf{w}}_{k}=\frac{1}{N_{i}} \sum_{n=1}^{N_{i}} \mathbf{w}_{k, n}
$$

has a chi-square distribution with $2 N_{i}$ degrees of freedom. When the number of samples $N_{i}$ is large, we can use the Central Limit Theorem to approximate $\overline{\mathbf{w}}_{k}$ as Gaussian [7], i.e., $\overline{\mathbf{w}}_{k} \sim N\left(E\left(\mathbf{w}_{k, n}\right), \boldsymbol{\Sigma}_{k} / N_{i}\right)$, where the variance matrix $\boldsymbol{\Sigma}_{\boldsymbol{k}}=\operatorname{diag}\left(\operatorname{Var}\left(\mathbf{w}_{k, n}(1)\right), \ldots, \operatorname{Var}\left(\mathbf{w}_{k, n}\left(\frac{N}{\gamma_{k}}\right)\right)\right)$. Therefore, for the $K$ sampling rates,

$$
\left[\begin{array}{c}
\overline{\mathbf{w}}_{1} \\
\vdots \\
\overline{\mathbf{w}}_{K}
\end{array}\right]=\left[\begin{array}{c}
\boldsymbol{\Phi}_{1} \\
\vdots \\
\boldsymbol{\Phi}_{K}
\end{array}\right]\left[\begin{array}{c}
\bar{P}_{s, 1} \\
\vdots \\
\bar{P}_{s, N}
\end{array}\right]+\mathbf{n}
$$

Denote $\overline{\mathbf{w}}^{C S}=\left[\begin{array}{c}\overline{\mathbf{w}}_{1} \\ \vdots \\ \overline{\mathbf{w}}_{K}\end{array}\right], \boldsymbol{\Phi}^{C S}=\left[\begin{array}{c}\mathbf{\Phi}_{1} \\ \vdots \\ \mathbf{\Phi}_{K}\end{array}\right], \mathbf{s}^{C S}=$ $\left[\begin{array}{c}\bar{P}_{s, 1} \\ \vdots \\ \bar{P}_{s, N}\end{array}\right]$, leading to the compact form

$$
\overline{\mathbf{w}}^{C S}=\boldsymbol{\Phi}^{C S} \mathbf{s}^{C S}+\mathbf{n}
$$




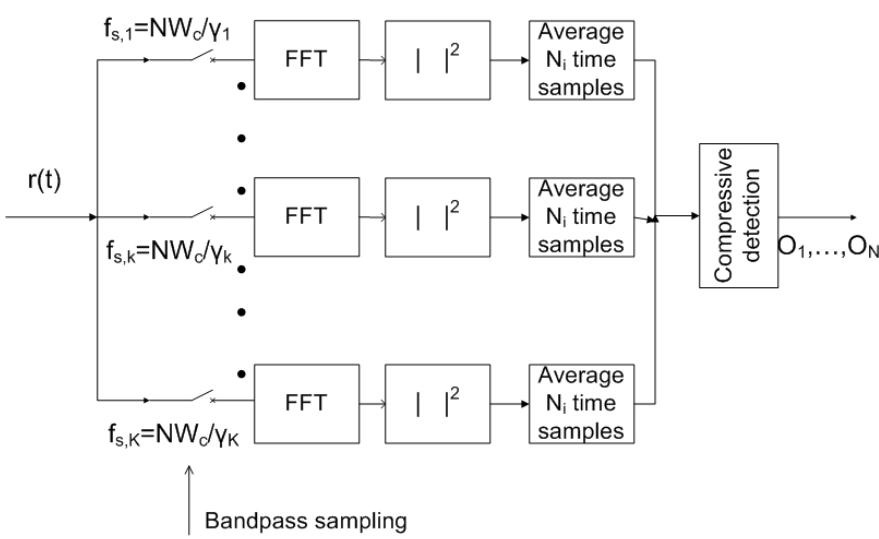

Fig. 6. Block diagram of the compressive detection scheme with bandpass sampling

where

$$
\boldsymbol{\Phi}^{C S}=\left[\begin{array}{ccccccc}
1 & 2 & 3 & 4 & 5 & \ldots & n=1, \ldots, N \\
1 & 0 & 0 & 0 & 0 & \ldots \\
0 & 1 & 0 & 0 & 0 & \ldots \\
\vdots & & & & & \\
1 & 0 & 0 & 0 & 0 & \ldots \\
\vdots & & & & & \\
0 & 0 & 0 & 0 & 0 & \ldots
\end{array}\right] \begin{gathered}
\left(n \bmod \frac{N}{\gamma_{1}}\right)=1 \\
\left(n \bmod \frac{N}{\gamma_{1}}\right)=2 \\
\vdots \\
\left(n \bmod \frac{N}{\gamma_{2}}\right)=1 \\
\vdots \\
\left(n \bmod \frac{N}{\gamma_{K}}\right)=0
\end{gathered}
$$

Define $\frac{N}{\gamma_{0}}=0$, then $\boldsymbol{\Phi}^{C S}(i, j)=1$, if $\exists 0 \leq l \leq K-$ 1, s.t. $\sum_{k=0}^{l} \frac{N}{\gamma_{k}}<i \leq \sum_{k=0}^{l+1} \frac{N}{\gamma_{k}}$, and $j \bmod \frac{N}{\gamma_{l+1}}=i-$ $\sum_{k=0}^{l} \frac{N}{\gamma_{k}}$; otherwise $\boldsymbol{\Phi}^{C S}(i, j)=0$.

The additive noise $\mathbf{n} \sim N\left(\boldsymbol{\Phi}^{C S}\left[\begin{array}{c}\bar{P}_{v, 1} \\ \vdots \\ \bar{P}_{v, N}\end{array}\right], \boldsymbol{\Sigma}^{C S}\right) . \mathrm{Be}-$ cause $\overline{\mathbf{w}}_{k_{1}}$ and $\overline{\mathbf{w}}_{k_{2}}$ are derived from samples from different time slots, $\forall k_{1} \neq k_{2}, \operatorname{Cov}\left(\overline{\mathbf{w}}_{k_{1}}\left(i_{1}\right), \overline{\mathbf{w}}_{k_{1}}\left(i_{2}\right)\right) \approx 0, \forall i_{1}, i_{2}$, yielding $\boldsymbol{\Sigma}^{C S} \approx \frac{1}{N_{i}} \operatorname{diag}\left(\boldsymbol{\Sigma}_{1}, \ldots, \boldsymbol{\Sigma}_{K}\right)$.

Because $\operatorname{Pr}\left(\bar{P}_{s, i}=0\right)=p_{i}$, the average number of nonzero elements in $\mathbf{s}^{C S}$ is $(1-\bar{p}) N$. It is proved in [28] that if $(1-\bar{p}) \ll 1$, and $\frac{N}{\gamma_{1}}, \ldots, \frac{N}{\gamma_{K}}$ are the first $K \sim O((N-$ $\left.\bar{p} N) \log _{N-\bar{p} N} N\right)$ prime numbers with $(1-\bar{p}) N \leq \frac{N}{\gamma_{1}}<$ $\ldots<\frac{N}{\gamma_{K}}$, then $\boldsymbol{\Phi}^{C S}$ satisfies RIP. Note that a lower bound for $\bar{p}$ is required for choosing $K$, since $\forall 0<p_{\text {low }}<p<1$, if $\boldsymbol{\Phi}^{C S}$ satisfies RIP for $\left(2\left(1-p_{\text {low }}\right) N, \delta_{2 N\left(1-p_{\text {low }}\right)}\right)$, it satisfies RIP for $\left(2(1-p) N, \delta_{2 N\left(1-p_{\text {low }}\right)}\right)$. Detection of the channel status $\mathbf{O}=\left[O_{1}, \ldots, O_{N}\right]^{T}$ in (4) is done by first solving for the signal powers from the following convex optimization problem

$$
\begin{array}{r}
\text { minimize }\|\hat{\mathbf{s}}\|_{1} \\
\text { subject to }\left\|\overline{\mathbf{w}}^{C S}-\boldsymbol{\Phi}^{C S} \hat{\mathbf{s}}\right\|_{2} \leq \epsilon
\end{array}
$$

where $\operatorname{Pr}\left(\|\mathbf{n}\|_{2} \leq \epsilon\right) \geq \lambda, \lambda \approx 1$. Then each element in $\hat{\mathbf{s}}=\left[\hat{P}_{s, 1}, \ldots, \hat{P}_{s, N}\right]^{T}$ is compared to a power threshold $\beta$ to determine if the channel is occupied, i.e., $\hat{P}_{s, i}>\beta \Rightarrow \hat{O}_{i}=$ 0 .

\section{PERFormance AnAlysis}

In this section, the performance of the proposed compressive spectrum sensing architecture with bandpass sampling is analyzed. The mean detection time and the storage required for the observation matrix is compared with the non-uniform sampler and the random pre-integrator in Table I.

\section{A. Mean Detection Time and Computational Complexity}

The mean detection time for the proposed compressive sensing architecture consists of three parts: integration time, time for switching ADC sampling rates, and latency for computation of the spectrogram. Since $N_{i}$ samples are used in determining status for each folded channel in $\overline{\mathbf{w}}_{k}$, the total number of samples needed equals $N_{i} N / \gamma_{k}$. Hence, the total integration time for the $k^{t h}$ sampling rate is $\frac{N_{i} N}{\gamma_{k}} \frac{\gamma_{k}}{N W_{c}}=$ $N_{i} / W_{c}$ and for the entire sensing process is $K N_{i} / W_{c}$. Denote the switching time between two different sampling rates as $T_{s w}$. The spectrogram $\overline{\mathbf{w}}_{k}$ for each sampling rate can be generated during the analog circuit switching time, because the FFT size for each sampling rate is $\frac{N}{\gamma_{k}} \ll N, k=1, \ldots, K$, and the signal processing time for FFT and averaging is negligible compared to $T_{s w}$. Therefore, the major component of the latency for computation of $\hat{\mathbf{s}}$ is the signal processing time $T_{l 1}$ required by (21). Thus the mean detection time for the proposed sensing scheme is

$$
T_{d, b p s}=K\left(N_{i} / W_{c}+T_{s w}\right)+T_{l 1}
$$

The number of rows $M$ of $\boldsymbol{\Phi}^{C S}$ in (20) is $K C_{L}$, where $C_{L}$ is the average of the first $K$ prime numbers no smaller than $N(1-\bar{p})$. In [28], it is proved that $C_{L} \sim O((N)(1-$ $\left.\bar{p}))\left(\log _{N-\bar{p} N} N\right) \ln \left((N-\bar{p} N) \log _{N-\bar{p} N} N\right)\right)$. As $K \sim O((N-$ $\left.\bar{p} N) \log _{N-\bar{p} N} N\right), M \sim O\left(C_{L}(N-\bar{p} N) \log _{N-\bar{p} N} N\right) . l_{1} \min -$ imization is applied to compute the averaged power spectrum directly, instead of the individual amplitude spectra for each snapshot. Thus the computational complexity associated with (21) is $O\left(M^{2} N^{1.5}\right)$, independent of $N_{i}$, leading to shorter signal processing time compared to the non-uniform sampler and the random pre-integrator.

Fig.7 illustrates the mean detection time for serial search with the heterodyne architecture, non-uniform sampler, random pre-integrator, and the proposed bandpass sampling architecture with $W_{c}=200 \mathrm{kHz}, T_{s w}=50 \mu \mathrm{s}$, and $N_{i}=1000$. As shown in [23], $T_{l 1} \approx((1-\bar{p}) N)^{3} \mu s . \bar{p}$ is chosen to be 0.9 so that the spectrum satisfies the sparsity requirement of CS. The results show that the bandpass sampling architecture consistently outperforms the serial search scheme for heterodyne architecture, by reducing analog circuit switching steps. For the bandpass sampling architecture, as $N$ increases, an increased number of sampling rates is required, leading to the increased mean detection time. For the non-uniform sampler and the random pre-integrator, as $N$ increases, the digital signal processing time becomes the major component in mean detection time, where $l_{1}$ minimization is applied to reconstruct the amplitude spectra and is executed $N_{i}$ times. When the signal processing duration is longer than the circuit switching time in the bandpass sampling architecture, the mean 


\begin{tabular}{|c||c|c|c|}
\hline & Non-uniform sampler & Random pre-integrator & Bandpass sampling \\
\hline Mean detection time & $N_{i} / W_{c}+N_{i} T_{l 1}$ & $N_{i} / W_{c}+N_{i} T_{l 1}$ & $K\left(N_{i} / W_{c}+T_{s w}\right)+T_{l 1}$ \\
Nyquist rate analog component & Yes & Yes & \\
Storage (projection matrix) & $O((N-\bar{p} N) \log (1 /(1-\bar{p})))$ & $O(N(N-\bar{p} N) \log (1 /(1-\bar{p})))$ & $O\left((N-\bar{p} N) \log { }_{N}-\bar{p} N N\right)$ \\
\hline
\end{tabular}

TABLE I

COMPARISON BETWEEN CS-BASED ARCHITECTURES

detection time of the non-uniform sampler and the random preintegrator exceeds that of the bandpass sampling architecture, because $K \ll N$.

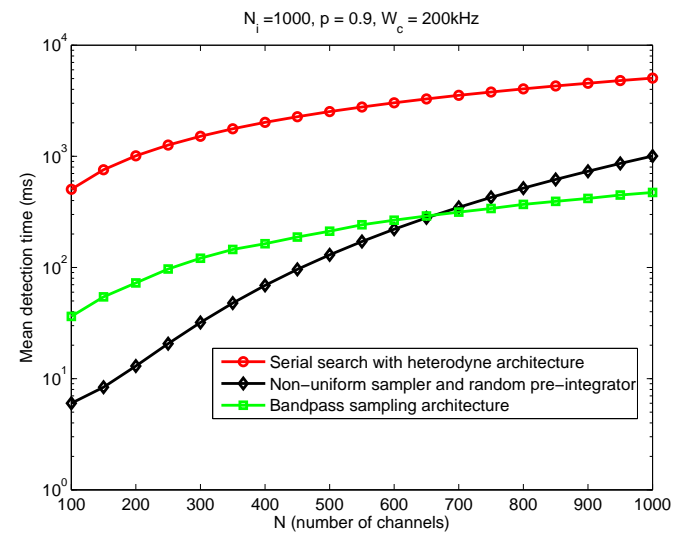

Fig. 7. Mean detection time of the serial search, non-uniform sampler, random pre-integrator and the proposed bandpass sampling architecture

In terms of storage requirements, only the sub-sampling factors $\gamma_{k}, k=1, \ldots, K$ need to be stored in memory, and the matrix $\boldsymbol{\Phi}^{C S}$ can be generated on-line according to (20). The storage required is smaller than the non-uniform sampler and the random pre-integrator.

\section{B. Detection performance and receiver operating character- istics}

The detection performance is influenced by the average SNR of all the channels. Assume a known signal is used for calibration, where the average SNR of the occupied channels is used for performance analysis. Denote the support of $\mathbf{s}^{C S}$ as $\Lambda=\left\{i: \bar{P}_{s, i}>0\right\}$. The input SNR is given by

$$
I S N R=\frac{\sqrt{\left(\sum_{i \in \Lambda} \bar{P}_{s, i}\right)^{2}}}{\sqrt{\left(\sum_{i \in \Lambda} \bar{P}_{v, i}\right)^{2}}}=C_{0} \frac{\left\|\mathbf{s}^{C S}\right\|_{2}}{\epsilon}
$$

where $C_{0}$ is determined by $N$ and $\bar{p}$. The postreconstruction SNR is given by

$$
R S N R=\frac{\left\|\mathbf{s}^{C S}\right\|_{2}}{\left\|\mathbf{s}^{C S}-\hat{\mathbf{s}}\right\|_{2}}
$$

where $\hat{\mathbf{s}}$ is output from (21) and $\mathbf{s}^{C S}$ consists of the actual signal powers. It is proved in [11] that with probability $\lambda \sim 1$, $\left\|\hat{\mathbf{s}}-\mathbf{s}^{C S}\right\|_{2} \leq C_{1} \epsilon$, where $C_{1}=\frac{2 \alpha}{1-\rho}, \rho=\frac{\sqrt{2} \delta_{2 \bar{L}}}{1-\delta_{2 \bar{L}}}, \alpha=$ $\frac{2 \sqrt{1+\delta_{2 \bar{L}}}}{\sqrt{1-\delta_{2} \bar{L}}}$, and $\bar{L}=(1-\bar{p}) N$ [11]. Denote $C=C_{0} C_{1}$. Therefore,

$$
I S N R \leq C \times R S N R
$$

The post-reconstruction SNR is $C$ times worse than the input SNR. Denote $P_{d}$ and $P_{f a}$ as detection rate and false alarm rate, respectively. Since $P_{d}-P_{f a}$ performance is proportional to RSNR and RSNR is bounded at least by a function of $\delta_{2 \bar{L}}$ and ISNR in (25), the detection rate $P_{d}$ and the false alarm rate $P_{f a}$ are determined by $\delta_{2 \bar{L}}$ and ISNR, where $\delta_{2 \bar{L}} \sim O\left(\frac{2 N(1-\bar{p})}{K} \log _{2 N(1-\bar{p})} N\right)$ according to [28].

To gain additional insight into the impact of $\delta_{2 \bar{L}}$ on the detection performance, the overall receiver operating characteristics for the bandpass sampling architecture are shown in Fig.8(a) and Fig.8(b), with $\bar{p}=0.95, N=100, N_{i}=1000$, and $p_{i}=\bar{p}, i=1, \ldots, N$. For $K=3$, the sampling rates used are $5 W_{c}, 7 W_{c}, 11 W_{c}$, and for $K=4$, an additional sampling rate $13 W_{c}$ is used, where all the sampling rates are smaller than the Nyquist rate $100 W_{c} . \bar{P}_{s, i}$ are set to 10 and $\bar{P}_{v, i}=1, i=1, \ldots, N$, so ISNR $=10 \mathrm{~dB}$. The numerical results of the estimated detection rate $\hat{P}_{d}$ and the estimated false alarm rate $\hat{P}_{f a}$ are averaged over 500 iterations. In each iteration, the locations of the occupied channels are different. As shown in Fig.8(a) and Fig.8(b), the performance for 4 sampling rates is better than 3 sampling rates, because $\delta_{2 N(1-\bar{p})}$ for $K=4$ is smaller than for $K=3$, yielding smaller $C$ in (25).

The impact of the ISNR $=\bar{P}_{s, i} / \bar{P}_{v, i}$ on the $P_{d}-P_{f a}$ performance is shown in Fig.9. As the ISNR decreases towards $9 \mathrm{~dB}, \hat{P}_{d}$ with 4 sampling rates $\left(5 W_{c}, 7 W_{c}, 11 W_{c}\right.$, and $\left.13 W_{c}\right)$ begins to decrease rapidly, because the spectrum of $\mathbf{R}$ is no longer sparse for $\boldsymbol{\Phi}^{C S}$ with the chosen sampling rates. In contrast, all the occupied channels are correctly detected for the 5 sampling rates $\left(5 W_{c}, 7 W_{c}, 11 W_{c}, 13 W_{c}\right.$, and $\left.17 W_{c}\right)$ over the 500 iterations for the SNR range shown in Fig. 9. Therefore, for a given ISNR, the detection performance of the proposed architecture can be improved by increasing the number of sampling rates, which imposes a trade-off between the detection performance and the detection time. The impact of the sparsity level of the spectrum is shown in Fig.10. For a smaller $\bar{p}$, an increased number of sampling rates is required for $\boldsymbol{\Phi}^{C S}$ to satisfy the RIP requirement as discussed in Sec.III, leading to a longer detection time.

\section{EXPERIMENTAL RESULTS}

We conducted a performance evaluation of our bandpass sampling scheme using the (publicly available) measurements of the power spectrum taken in a modern office building in Aachen, Germany, in 2007 [29]. The spectral band observed is $20 \mathrm{MHz}-1.64 \mathrm{GHz}$ (center frequency $770 \mathrm{MHz}$ ). The RF signal was amplified, down-converted to the base band, and 


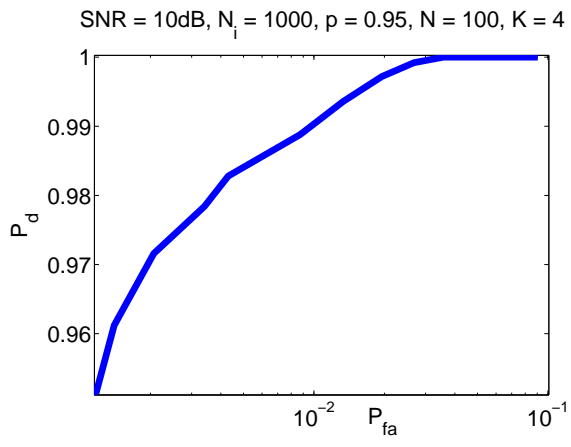

(a) $K=4$

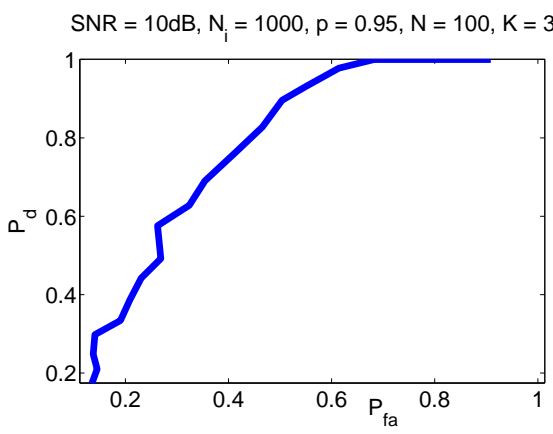

(b) $K=3$

Fig. 8. Receiver operating characteristics for the proposed bandpass sampling architecture with $K$ sampling rates

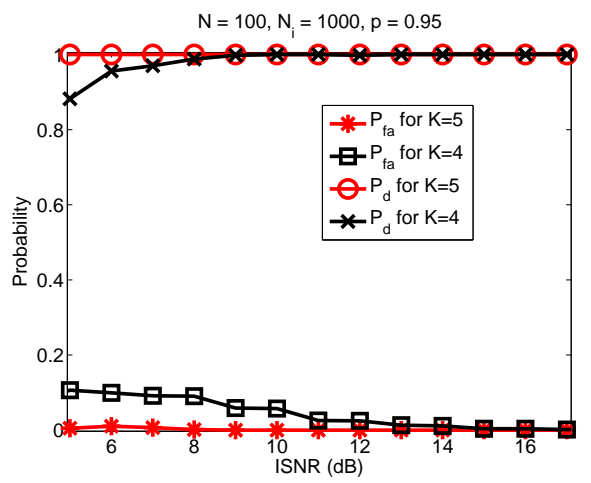

Fig. 9. Impact of the SNR on the $P_{d}-P_{f a}$ performance

processed by an Agilent E4440A spectrum analyzer. The analyzer produces a 8192-point FFT on the signal, where each FFT bin represents a $200 \mathrm{kHz}$ resolution width. The magnitude squared FFT value represents the instantaneous spectrogram (power spectrum) over a channel. A full scan of the 8192 channels requires about 1.8 second. The average noise level of the receiver chain is $-169 \mathrm{dBm}$ at $1 \mathrm{~Hz}$ resolution bandwidth, which is $-116 \mathrm{dBm}$ for the $200 \mathrm{kHz}$ channel. The band plan for spectrum usage in Germany was not available, so the channel status is first estimated by thresholding the original data at $-111 \mathrm{dBm}$, corresponding to constant false alarm rate of $10^{-5}$. The data from the final 100 channels at $12: 37 \mathrm{am}, 2: 37 \mathrm{am}$, $4: 37 \mathrm{am}, 6: 37 \mathrm{am}$, and $8: 37 \mathrm{am}$ on Jan.27th, is used as input for the bandpass sampling architecture.

Among the 100 channels, two channels are occupied as shown in Fig.11(a) and Fig.11(b). For the bandpass sampling

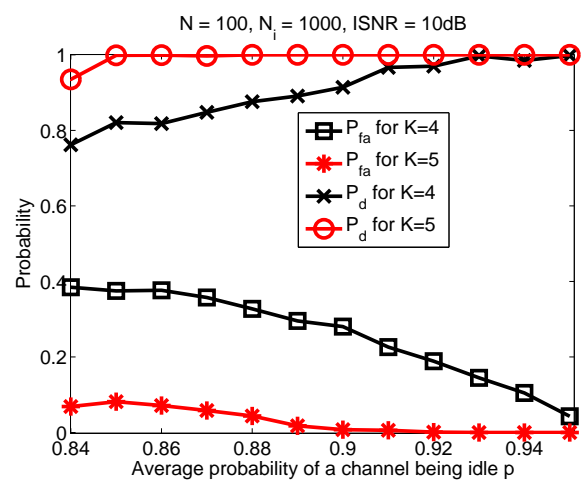

Fig. 10. Impact of the sparsity level $\bar{p}$ on the $P_{d}-P_{f a}$ performance

architecture, the 4 sampling rates used are $5 W_{c}, 7 W_{c}, 11 W_{c}$, and $13 W_{c}$, and for 5 sampling rates, $17 W_{c}$ is added. To enhance signal sparsity, the average noise level of $-116 \mathrm{dBm}$ is removed from the original data, and then added back in the reconstructed power spectrum for comparison with the original spectrum. The proposed architecture with $K=4$ achieves a $\hat{P}_{d}=1$ while $\hat{P}_{f a}=0.01$ for all the 5 time slots, with the same threshold of $-111 \mathrm{dBm}$. With $K=4$, the reconstructed power spectrum for $12: 37 \mathrm{am}$ and $8: 37 \mathrm{am}$ is shown in Fig.11(a) and Fig.11(b). Although the SNR of the reconstructed signal is worse than the original signal, both of the 2 occupied channels can be accurately identified, but a false alarm occurs in Fig.11(b). As shown in Fig. 11(c), with $K=5$, no false alarm occurs for $8: 37 \mathrm{am}$, because the reconstructed SNR is improved according to Section IV.

\section{CONCLUSION}

A compressive spectrum sensing architecture with bandpass sampling is proposed to detect idle channels in cognitive radio. The bandpass sampling technique enables sub-Nyquist ADCs and simplifies the receiver circuits design. Compared to other compressive sensing architecture, the proposed architecture does not have any Nyquist rate analog circuit components. Numerical results show that the proposed algorithm achieves lower mean detection time compared to the serial search scheme when the channel occupancy rate is low and is a promising alternative when Nyquist rate ADC is not feasible.

\section{REFERENCES}

[1] J. Ma, G.Y. Li, and B. H. Juang. Signal processing in cognitive radio. Proceedings of the IEEE, 97(5):805 -823, May 2009.

[2] A.A. Abidi. Direct-conversion radio transceivers for digital communications. Solid-State Circuits, IEEE Journal of, 30(12):1399-1410, Dec. 1995.

[3] B. Razavi. RF microelectronics. Prentice Hall communications engineering and emerging technologies series. Prentice Hall PTR, 1998.

[4] S. J. Shellhammer. Spectrum sensing in ieee 802.22. IAPR Wksp. Cognitive Info. Processing, Jun. 2008.

[5] L. Luo, N.M. Neihart, S. Roy, and D.J. Allstot. A two-stage sensing technique for dynamic spectrum access. Wireless Communications, IEEE Transactions on, 8(6):3028 -3037, Jun. 2009.

[6] S. Pollin, L. Hollevoet, P. Van Wesemael, M. Desmet, A. Bourdoux, E. Lopez, F. Naessens, P. Raghavan, V. Derudder, S. Dupont, and A. Dejonghe. An integrated reconfigurable engine for multi-purpose sensing up to $6 \mathrm{ghz}$. In New Frontiers in Dynamic Spectrum Access Networks (DySPAN), 2011 IEEE Symposium on, pages 656 -657, May 2011. 
[7] D. Cabric, A. Tkachenko, and R. W. Brodersen. Experimental study of spectrum sensing based on energy detection and network cooperation. In Proceedings of the first international workshop on Technology and policy for accessing spectrum, TAPAS '06, New York, NY, USA, 2006.

[8] Z.H. Derafshi, J. Frounchi, and H. Taghipour. A high speed fpga implementation of a 1024-point complex fft processor. In Computer and Network Technology (ICCNT), 2010 Second International Conference on, pages $312-315$, april 2010.

[9] I. F. Akyildiz, W. Lee, M. C. Vuran, and S. Mohanty. Next generation/dynamic spectrum access/cognitive radio wireless networks: A survey. Computer Networks, 50(13):2127 - 2159, 2006

[10] D.L. Donoho. Compressed sensing. Information Theory, IEEE Transactions on, 52(4):1289 -1306, Apr. 2006.

[11] E. J. Candes. The restricted isometry property and its implications for compressed sensing. Comptes Rendus Mathematique, 346(9-10):589592, 2008.

[12] Z. Tian. Compressed wideband sensing in cooperative cognitive radio networks. In Global Telecommunications Conference, IEEE, pages 1 -5, Dec. 2008.

[13] M. Mishali and Y.C. Eldar. Wideband spectrum sensing at subnyquist rates [applications corner]. Signal Processing Magazine, IEEE, 28(4):102 -135, July 2011.

[14] J. Meng, W. Yin, H. Li, E. Hossain, and Z. Han. Collaborative spectrum sensing from sparse observations in cognitive radio networks. Selected Areas in Communications, IEEE Journal on, 29(2):327 -337, february 2011.

[15] J. P. Elsner, M. Braun, H. Jakel, and F. K. Jondral. Compressed spectrum estimation for cognitive radios. Proceedings of 19th Virginia Tech Symposium on Wireless Communications, pages 1-4, June 2009.

[16] E. J. Candes and M. B. Wakin. People hearing without listening: an introduction to compressive sampling. IEEE Signal Processing Magazine, 25(2):21 -30, march 2008.

[17] M. Mishali, Y.C. Eldar, O. Dounaevsky, and E. Shoshan. Xampling: Analog to digital at sub-nyquist rates. Circuits, Devices Systems, IET, 5(1):8-20, Jan. 2011.

[18] J. Laska, S. Kirolos, Y. Massoud, R. Baraniuk, A. Gilbert, M. Iwen, and M. Strauss. Random sampling for analog-to-information conversion of wideband signals. In Design, Applications, Integration and Software, 2006 IEEE Dallas/CAS Workshop on, pages 119 -122, Oct. 2006.

[19] M. Mishali and Y.C. Eldar. Blind multiband signal reconstruction: Compressed sensing for analog signals. Signal Processing, IEEE Transactions on, 57(3):993 -1009, Mar. 2009.

[20] Q. Hui and Y. Lun. Bandpass sampling for multiband signal on exponent demodulation function. In Mobile Congress (GMC), 2010 Global, pages $1-4$, Oct. 2010

[21] J.A. Tropp, J.N. Laska, M.F. Duarte, J.K. Romberg, and R.G. Baraniuk. Beyond nyquist: Efficient sampling of sparse bandlimited signals. Information Theory, IEEE Transactions on, 56(1):520 -544, Jan. 2010.

[22] A. Y. Yang, A. Ganesh, Z. Zhou, S. Sastry, and Y. Ma. A review of fast 11-minimization algorithms for robust face recognition. CoRR, abs/1007.3753, 2010 .

[23] I. Loris. On the performance of algorithms for the minimization of 11 -penalized functionals. Inverse Problems, 25(3):035008, 2009.

[24] R.H. Walden. Analog-to-digital converter survey and analysis. Selected Areas in Communications, IEEE Journal on, 17(4):539 -550, Apr. 1999.

[25] D.M. Akos, M. Stockmaster, J.B.Y. Tsui, and J. Caschera. Direct bandpass sampling of multiple distinct rf signals. Communications, IEEE Transactions on, 47(7):983 -988, Jul. 1999.

[26] C. Ghosh, S. Roy, M. B. Rao, and D. P. Agrawal. Spectrum occupancy validation and modeling using real-time measurements. In Proceedings of the 2010 ACM workshop on Cognitive radio networks, CoRoNet '10, pages 25-30, New York, NY, USA, 2010.

[27] M. Abramowitz and I. A. Stegun. Handbook of Mathematical Functions with Formulas, Graphs, and Mathematical Tables. Dover, New York, ninth dover printing, tenth gpo printing edition, 1964.

[28] M.A. Iwen. Simple deterministically constructible rip matrices with sublinear fourier sampling requirements. In Information Sciences and Systems, 43rd Annual Conference on, pages 870 -875, Mar. 2009.

[29] M. Wellens and P. Mahonen. Lessons learned from an extensive spectrum occupancy measurement campaign and a stochastic duty cycle model. Mobile Networks and Applications, 15:461-474, 2010.

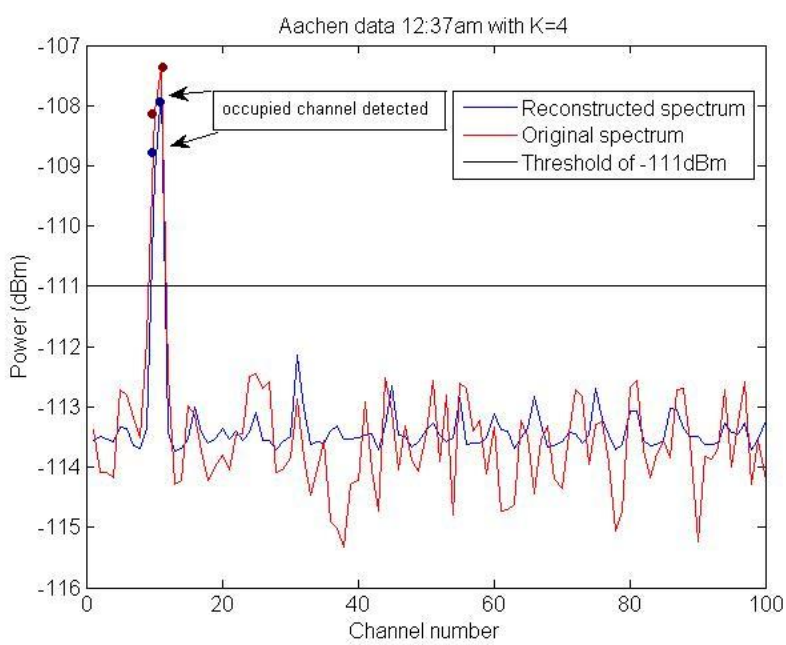

(a) The detection / estimation performance on 12:37am data with $\mathrm{K}=4$

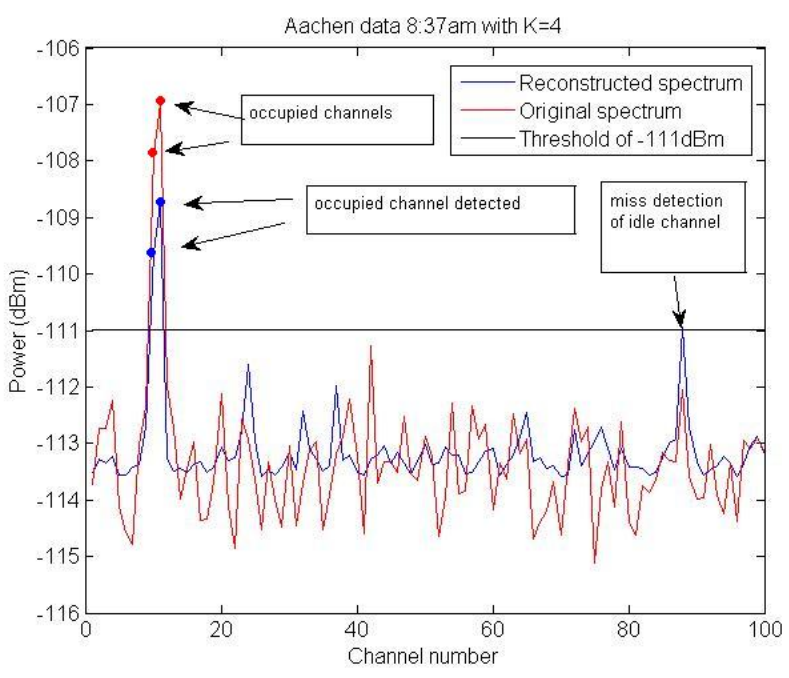

(b) The detection / estimation performance on 8:37am data with $\mathrm{K}=4$

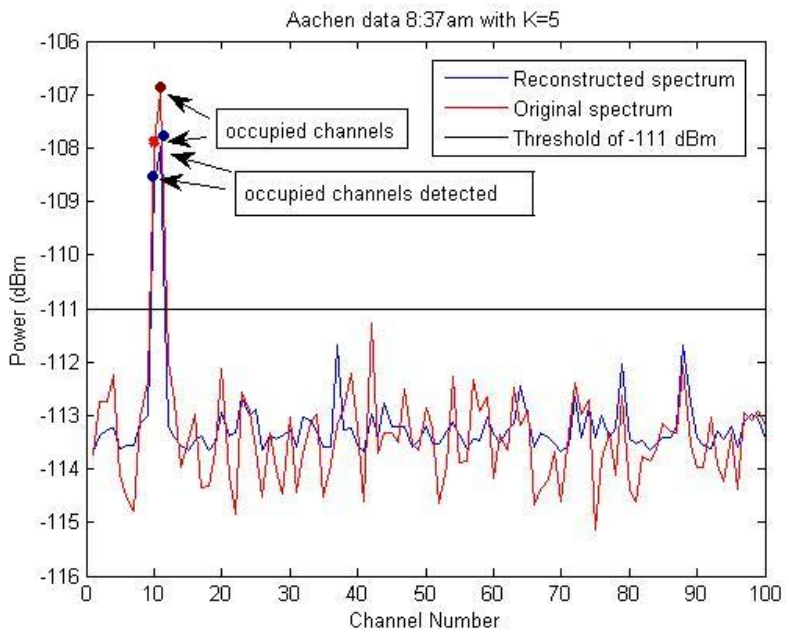

(c) The detection / estimation performance on 8:37am data with $\mathrm{K}=5$

Fig. 11. Performance of the bandpass sampling architecture on final 100 channels of the Aachen dataset on Jan.27th 